\title{
Povidone Iodine (PVP-I) Oro-Nasal Spray: An Effective Shield for COVID-19 Protection for Health Care Worker (HCW), for all
}

\author{
Mostafa Kamal Arefin ${ }^{1}$ iD
}

Received: 8 February 2021/Accepted: 22 March 2021/Published online: 8 April 2021

(C) Association of Otolaryngologists of India 2021

\begin{abstract}
SARS- CoV-2 or novel coronavirus enters in human body through nose and mouth, stays there for a while. Then binds with ACE2 receptor, enters inside cell, multiply there and manifests. Again, Polyvinyl Pyrrolidone or Povidone Iodine (PVP-I) is a strong microbicidal agent having $99.99 \%$ virucidal efficacy in its only $0.23 \%$ concentration, irrespective of all known viruses, even in SARS- CoV-2 (in vitro). An oro-nasal spray is designed to apply the PVP-I in nose and oral cavity to gain a protective layer or coating over nasal and oral mucosa, so that SARSCoV-2 can't bind with the ACE-2 receptor and prevent their entry inside. So, it will be effective for prevention of COVID-19. Moreover, as PVP-I has the ability for destruction of SARS-CoV-2, transmission of SARS- CoV2 from patient will be reduced also. Thus PVP-I oro-nasal spray can act as an effective shield for COVID-19 protection for healthcare workers, for all.
\end{abstract}

Keywords PVP-I · Oro-nasal spray · COVID-19

$\begin{array}{ll}\text { Abbreviations } & \\ \text { PVP-I } & \text { Polyvinyl Pyrrolidone or Povidone Iodine } \\ \text { HCW } & \text { Health care worker } \\ \text { SARS-CoV-2 } & \begin{array}{l}\text { Severe Acute Respiratory Syndrome- } \\ \text { Corona Virus-2 }\end{array}\end{array}$

Mostafa Kamal Arefin

arefin61dmc@gmail.com

1 Department of ENT \& Head Neck Surgery, Dhaka Medical College \& Hospital, Dhaka, Bangladesh

\section{Background}

Coronavirus disease 2019 (COVID-19) has already affected millions of people with more than a million deaths worldwide since the advent of SARS-CoV-2 in late 2019 [1]. SARS- CoV-2 enters in human body through nose and mouth, stays there for a while. Then binds with ACE2 receptor, enters inside cell, multiply there and manifests. Again, it is already established that PVP-I is a strong microbicidal (chemical) agent, having $99.99 \%$ virucidal efficacy in its only $0.23 \%$ concentration, irrespective of all known viruses, even in SARS-CoV-2 (in vitro) [2, 3, 4].

The first step in the development of URTIs is the adherence and colonisation of the respiratory pathogen to the nasopharyngeal and oropharyngeal mucosa. Assuming nasal and oral entry of such pathogens, intranasal and intra oral application of Povidone Iodine offers a practical measure for their prevention.

In lots of articles of scholars from different part of the world, use of PVP-I for intranasal and /or oral application is proposed or recommended, for COVID-19 prevention and for reduction of transmissibility of COVID-19 [4, 5, 6, 7, 8]. Several articles of different doctors and scientists recommended for a clinical trial on intranasal or intraoral application of PVP-I.

Here, an effective and safe Oro-Nasal Spray is designed to apply the PVP-I in nose and oral cavity to gain a protective layer or coating over nasal and oral mucosa, so that SARS-CoV-2 can't bind with the ACE-2 receptor and prevent their entry inside. Thus it will be effective for prevention of COVID-19. Moreover, as PVP-I has the ability for destruction of SARS-CoV-2, transmission of SARS- CoV-2 will be reduced also. 


\section{Oro-Nasal Spray}

Nasal drop works on nasal mucosa, part of nasopharynx. It passes through the floor of the nose and large area of nasopharynx is escaped or not covered. Nasal drops are cleared more quickly from the nose than nasal sprays. Nasal drops are to be used usually in lying down position. Nasal spray is easier to use as it diffuses better and reaches deeper into the nose. Nasal spray can be used in sitting position as well. PVP-I can be reached in the wide area of nasopharynx, even furthest area effectively and successfully by using nasal spray. In these context, nasal spray is better than nasal drop for distributing the drug in deeper structure or difficult to reach structure and in nasopharynx.

In Japan, $0.45 \%$ PVP-I throat spray is being used since long for prevention of cold and sore throat treatment. Gargling with PVP-I or spraying the throat with PVP-I is encouraged and included in their national respiratory guideline $[9,10]$.

Again, PVP-I is being used as mouthwash throughout the world (, specially PVP-I 1\% mouthwash in Asia,) since long with safety and good efficacy. PVP-I $0.5 \%$ nasal drop or nasal spray is being proposed in multiple scientific articles $[4,5,6,7]$.

After getting positive or significant result ( yet not published) from a clinical trial of " Virucidal efficacy of PVP-I on SARS- CoV-2 in nasopharynx (NCT04549376), I am proposing $0.6 \%$ PVP-I Oro-Nasal spray is to be used as protective measures. In that trial, among 189 patients $0.6 \%$ PVP-I nasal spray showed better efficacy (,i.e. about $81.5 \%)$ than other $(0.4 \%, 0.5 \%$ PVP-I nasal spray) with almost no mucosal irritation.

I proposed (,made and started using) an Oro-Nasal Spray, which can be used in nose as well as throat. It's nothing but $0.6 \%$ PVP-I solution, kept in a simple nasal spray device. To prevent COVID-19 transmission from patient to $\mathrm{HCW}$, HCW (asymptomatic carrier) to common people, or patient of other disease, this proposed oro-nasal spray will definitely be helpful.

\section{Safety of PVP-I Oro-Nasal spray}

Oral PVP-I gargle, throat spray, nasal spray formulations are currently available as over-the-counter medications in many countries, including Japan and Canada. PVP-I in low concentrations has not been known to stain teeth or to cause any major adverse effect. PVP-I oronasal spray should better to avoid in patients with iodine allergy or those undergoing radioiodine treatment or thyroid dysfunction. In a study PVP-I is proved ciliotoxic in concentrations of $5 \%$ and $10 \%$, but not in proposed low concentration 8 .

The topical application of iodine (in low concentration) intranasally for the treatment of recalcitrant chronic rhinosinusitis has been described by the St. Paul's Sinus Centre team in Vancouver. They found it was beneficial for the management of this condition, but also did not lead to any significant effect on thyroid function, mucociliary clearance or olfaction 4,5 .

In a study conducted by Gluck et al. three groups received liposomal dispersion with PVP-I (2.2, 4.4 and $0 \%$ as control) in single and repeated use. Application of liposomal PVP-I spray to the nasal mucosa does not result in any demonstrable limitation of the nasal function nor in detectable damage to the multilayer ciliated epithelium of the nose. Improvement of various parameters of nasal function under liposomal PVP-I suggest improved mucociliary clearance. Explanation could be humidification, improved surfactant (phospholipid) level and/or sufficient mucolytic activity of iodide due to local application of the constituents $[4,5,11]$. So $0.6 \%$ PVP-I Oro-Nasal spray is safe for long time use Figs. 1, 2, 3, 4 and 5.

\section{My Experience with PVP-I in COVID-19 Era}

Like lot of doctors and scientists of different part of the world, I have firm belief on PVP-I, which is a time tested, trusted virucidal agent. More than 50 dedicated doctors (including me) and other HCW of my network voluntarily use this oro-nasal spray for same purpose for the last 5-9 months.

I personally use it by myself in my private practice, in all of my surgery, all of the OPD procedures. Irrespective of COVID-19 status, either RT-PCR test positive or

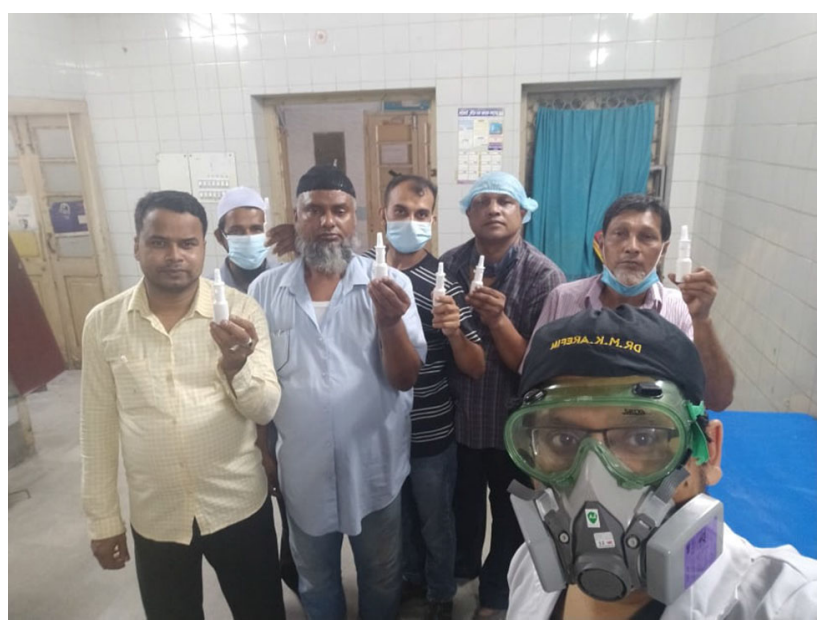

Fig. 1 HCWs of Dept. ENT \& HNS, DMCH holding the PVP-I OroNasal spray 


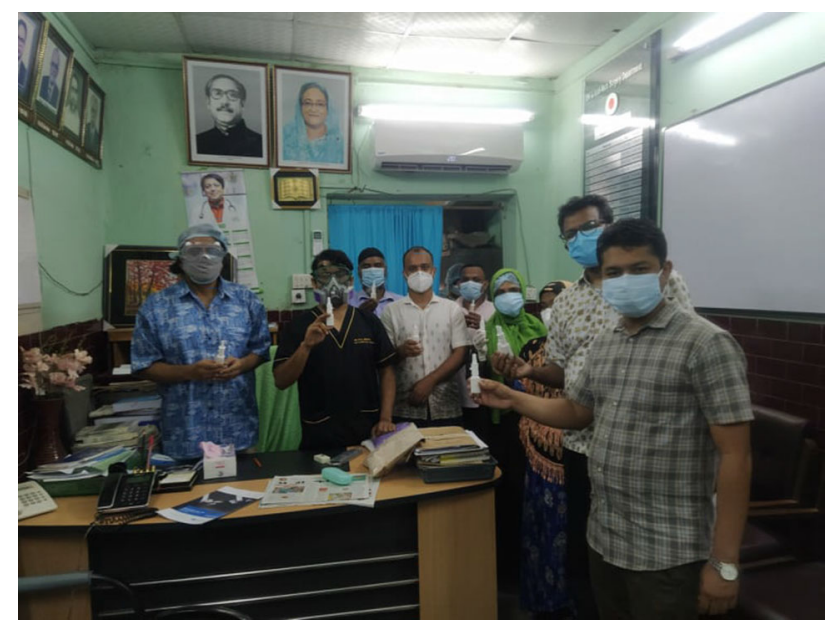

Fig. 2 Doctors and other HCWs of Dept. ENT \& HNS, DMCH holding the PVP-I Oro-Nasal spray

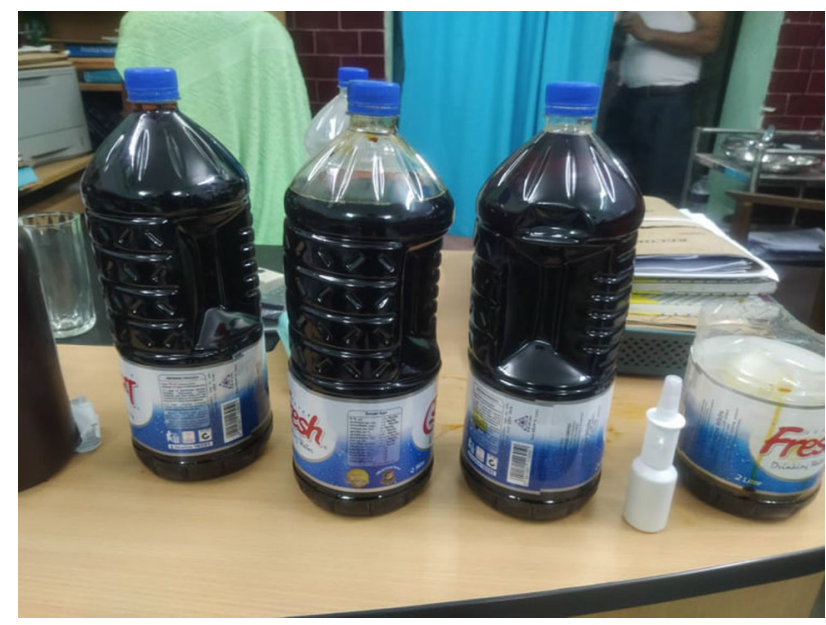

Fig. 3 PVP-I solutions prepared for gargling and Oro-nasal spray

negative. All of my staffs are trained enough to prepare my patients as well as themselves with PVP-I either by gargling/spraying their throat and/or spraying in nose prior to visit me. Since May 2020 I have attended more than 7000 patient in OPD and emergency, performed 501 endoscopy of larynx and nose (FOL, DNE) and 203 surgeries, including tracheostomy, mastoidectomy, endoscopic sinus surgery, skull base surgery, laryngeal biopsy, tonsillectomy, septoplasty etc. These are airway surgeries and moreover, most of them are aerosol generating procedures) $[12,13,14]$.

In my private practice I always examined thoroughly all the area of my concern (i.e. oral cavity, nose and ear) without hesitation, without compromising the quality of proper examination. My patients were always satisfied and compliance was extraordinarily good, because oral cavity and nose examinations are avoided mostly in COVID-19 era.

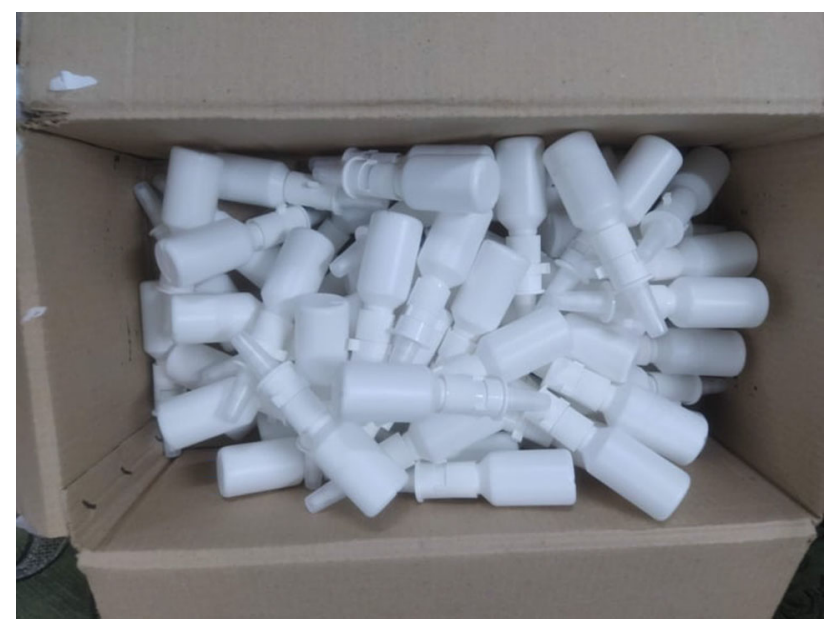

Fig. 4 Containers of PVP-I Oro-Nasal spray

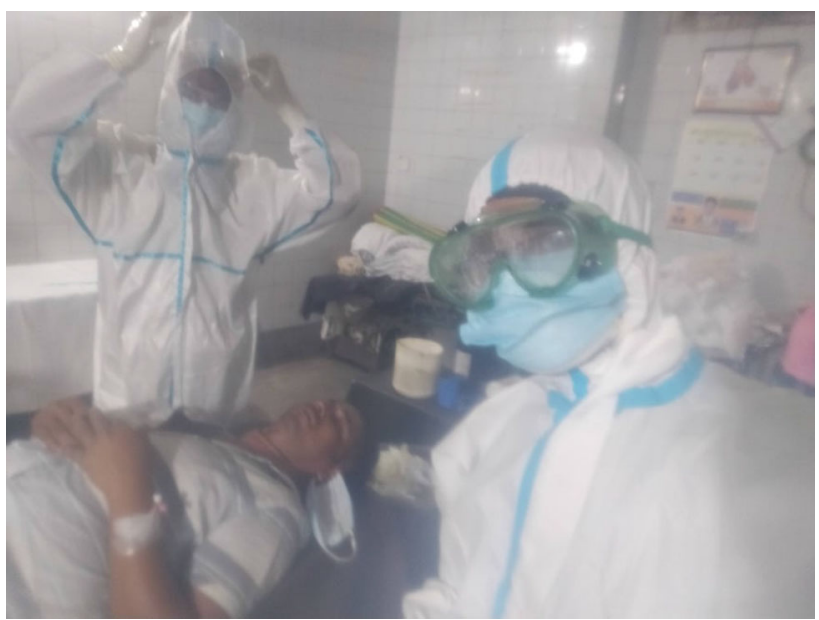

Fig. 5 During visiting patient in COVID unit of DMCH

Among all of my OPD patients more than 51 person diagnosed COVID-19 positive later, as I advised them to do RT-PCR test following physical examination and they were tested positive.

2 of my patients of emergency tracheostomy diagnosed COVID-19 positive 1 day later. Three nearby patients as well as two attendants were infected by them within one day of hospital stay (in non COVID unit). Here, I should mention that, Tracheostomy is one of the most aerosol generating procedure, where chance of COVID-19 transmissibility is more than any other surgery. Interestingly, among twelve different health care providers, including doctors, nurses and OT staffs, directly involved and exposed in these two surgeries, none of them were infected. Importantly, 'POLIDON' approach was followed in both the procedures $[12,14]$. 
For the previously mentioned clinical trial purpose, I had to go in close contact with COVID-19 patients for 31 days in the COVID-19 dedicated unit of Dhaka Medical College Hospital within 45 days. During nasal irrigation or spraying, patients cough on me or my team members several times. But for the grace of almighty Allah (God), me with all of my team members are free from COVID-19 still now.

HCWs of multiple hospitals or institutes of different parts of the world are using PVP-I in different form for protection from SARS-CoV-2. In Bangladesh authorities or individual doctors of Dhaka Medical College Hospital, Bangabandhu Sheikh Mujib Medical University, National Institute of ENT, Bangladesh ENT Hospital, ENT and Head Neck Cancer Hospital and most of the ENT, dental or maxillofacial related health care professionals adopted this policies in different extent.

Adjunct to PPE, mask, hand sanitizer, my strong belief in this regard-use of PVP-I oro-nasal spray gives me an extra protective layer over nasal, nasopharyngeal, oral and oropharyngeal mucosa. Moreover, when my patient gargles with PVP-I or spray PVP-I solution in oral cavity, throat and nose all the SARS- CoV-2 of these areas are destroyed and absent, at least for certain period of time. It reduces the possibility of SARS-CoV-2 transmission from patient, which made me more courageous and confident. Figures 6, 7, 8, 9 and 10

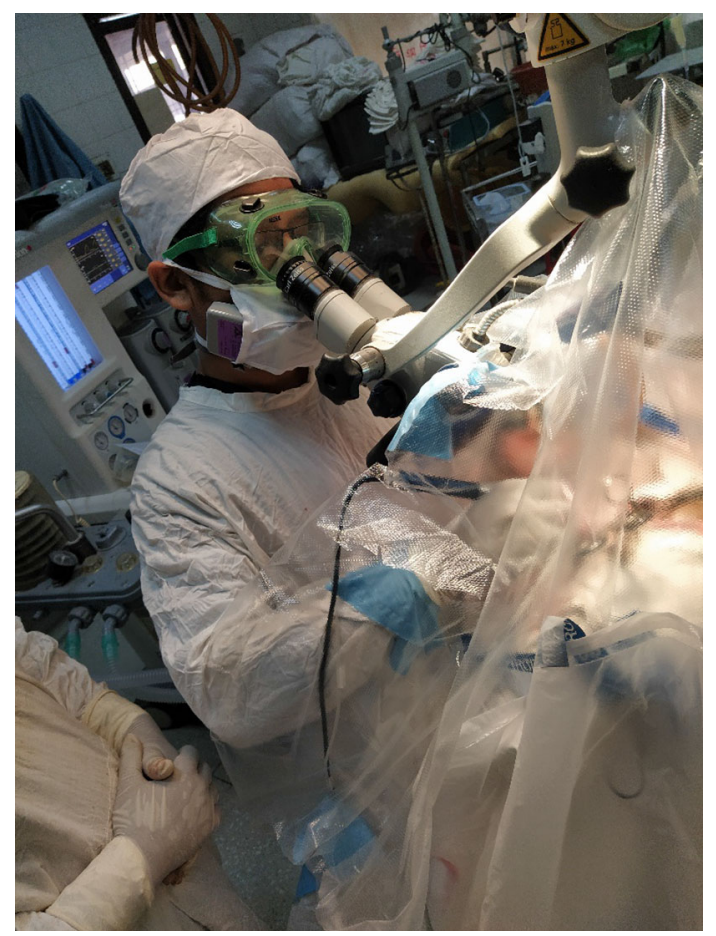

Fig. 6 During performing ear surgery (mastoidectomy) in 'POLIDON' approach (POLIDON = Polythene + Povidone Iodine)

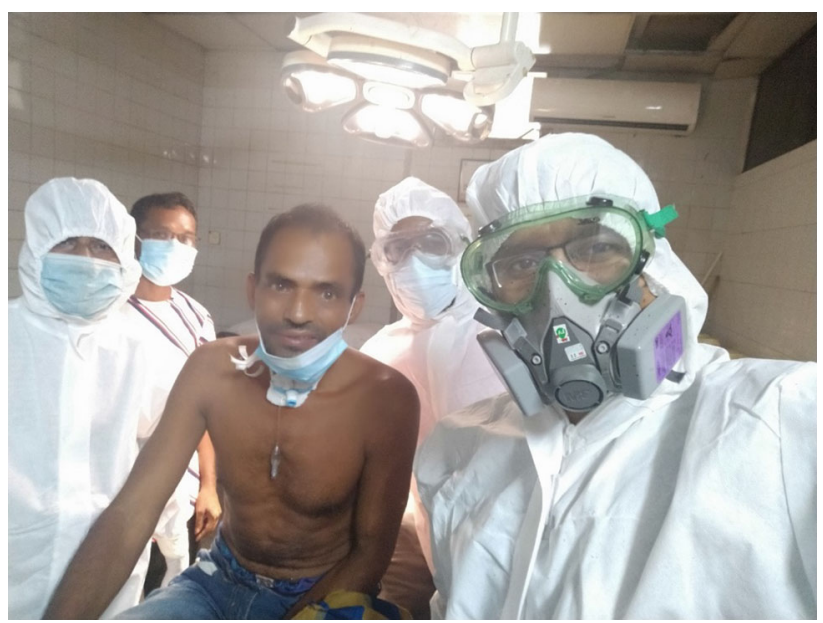

Fig. 7 Immediately after emergency tracheostomy

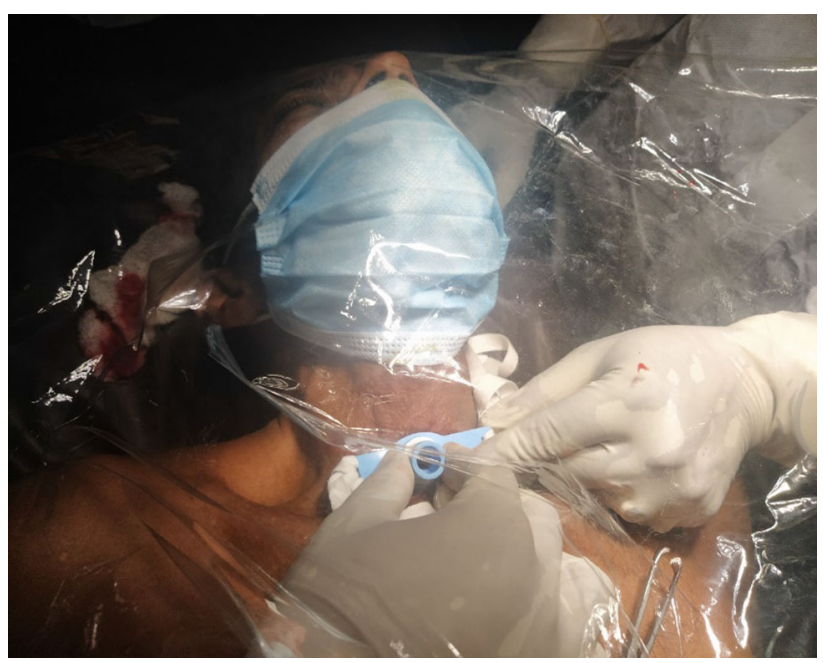

Fig. 8 During Emergency tracheostomy in COVID-19 pandemic (by 'POLIDON' approach)

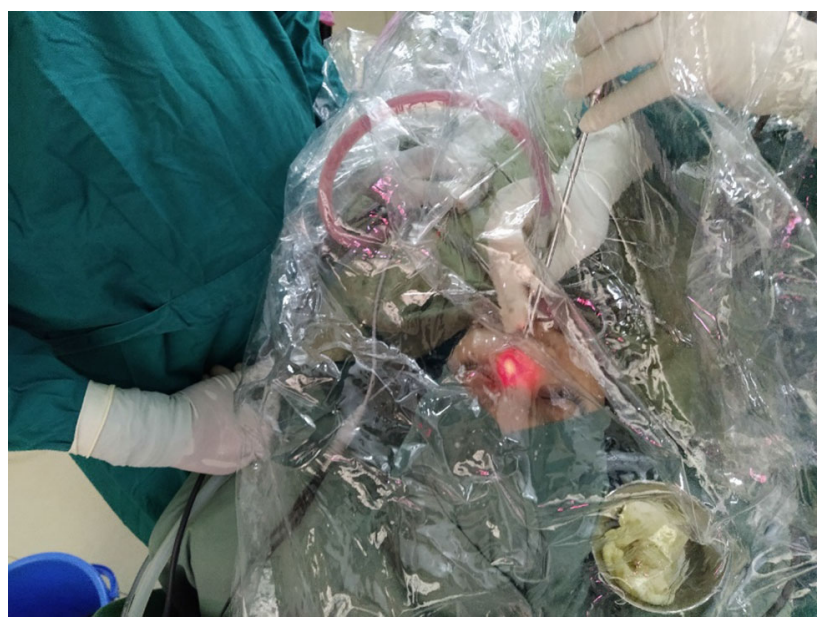

Fig. 9 During Endoscopic sinus surgery in COVID-19 era ('POLIDON' approach) 


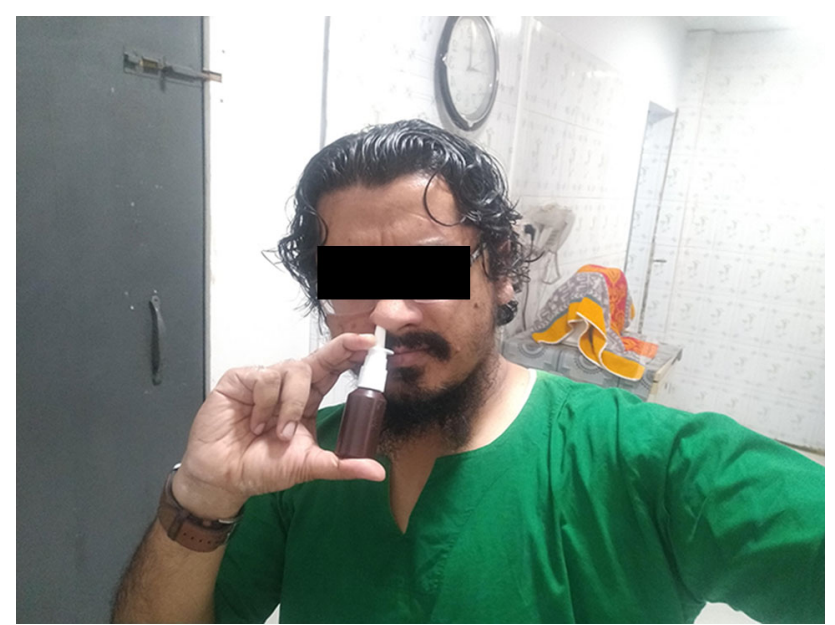

Fig. 10 Use of PVP-I Oro-Nasal spray (Post exposure). (N.B. Please ignore the label of container)

\section{Benefits of PVP-I Oro-Nasal Spray in Brief}

\section{Benefits of 0.6\% PVP-I}

- Effective virucidal agent against all coronaviruses, including SARS- COV-2.

- Effective in case of both pre and post exposure.

- Effective for healthy individual (for protection) as well as COVID-19 patient also ( for reduction of viral load and SARS-CoV-2 transmissibility for close contacts/ family members).

- Almost no side effect (mucosal irritation, thyroid dysfunction, teeth staining,).

- This minimum concentration of PVP-I (0.6\%) has least possibility of doing any harm to human body (nasal or oral mucosa). More concentration is being used on eye, ear, mucosa of oral cavity, oropharynx since before.

- No chance of resistance

- Reduces disease burden by minimizing sign- symptoms, like-nasal congestion, sore throat.

\section{Benefits of Oro-Nasal Spray}

- Single container, double function (PVP-I Oral/Throat spray as well as Nasal spray)

- Easy to carry (in pocket, handbag)

- Easy to use

- No need to have a basin or a facility to gargle or spit

- Minimum amount of PVP-I solution is needed $(20 \mathrm{ml}$ solution can be used for 20- 30 days for regular user.)

- Cheap (to refill)

- Minimum Iodine exposure.

- Container can be reusable and refillable.

\section{Recommendations}

PVP-I Oro Nasal Spray should be used by doctors or other health care workers (HCW), COVID-19 patients as well as common people.

\section{For Doctors or Other Health Care Workers (HCW).}

- Prior to visit any patient in any facility irrespective of COVID status

- Prior to examining the oral cavity, throat or nose of any patient

- Prior to any Surgery, specially surgery of Nose, mouth, throat, ear, skull base

- Prior to attend COVID-19 patient in a COVID-19 dedicated unit / hospital

- Following exposure to confirmed or suspected COVID patient (intentional/accidental)

\section{For Common People}

- Prior to attend public gathering or working outside home, where there is chance of known or unknown COVID-19 patient/ SARS-CoV-2 exposure.

- Before leaving and after returning home and in between 1 or 2 time (after tea/lunch/dinner break).

For COVID-19 patients

As it has the potentiality to reduce the viral load, it will definitely reduce the viral transmission from patient to healthy person. Again, it may have positive role on treatment, in mortality and morbidity index. To prove this further studies are recommended.

How to use?

2-3 puff in each nostril and 1-3 puff in throat (oral cavity and oropharynx) in 3-4 h interval.

How long?

Though it is proved and established that long term (1-2 months) application of this minimum amount of PVP-I is quite safe SPS:refid::bib2lbib3lbib4[2,3,4]. But, I recommend after continuous use of 1-2 week 1 day interval (weekly holiday) should be given. 


\section{Conclusion}

PPE, mask, hand sanitization or vaccines nothing proved $100 \%$ effective for COVID-19 prevention still now. PVP-I oro-nasal spray is an easy tool or way for COVID-19 prevention and reduction of SARS-CoV-2 transmissibility. I recommend here, this oro-nasal spray should be used as an adjunct to PPE, mask or vaccine. Any person, policy maker, local or central government could adopt this safe, cheap, easily available and effective PVP-I Oro-Nasal spray as an additional shield of their COVID-19 protection and thus minimize the COVID burden.

Acknowledgements I express my heartfelt thanks to Brig. Gen Dr. A K M Nasir Uddin (Ex-Director, Dhaka Medical College Hospital), Prof Dr. Shaikh Nurul Fattah Rumi- (Head of the Dept. of ENT \& Head Neck Surgery), Prof Dr. Sultana Sahana Banu (Head of Dept. of Virology), Dr. Mala Khan - the DG of BRICM, Asso Prof Dr. Debesh Chandra Talukder (ENT), Dr. Md. Obaidur Rahman (Radiology), Dr. Md. Ahsanul Kaiser- Post Doctoral Fellow of BCSIR (Nano Medicine \& Theranostics), Assistant Prof Dr. Muhammad Shaharior Arafat (ENT), Asst. Prof. Dr. Nusrat Sultana Leema (Virology), Dr. Joybaer Anam Chowdhury (Medicine), Dr. Md. Mamunur Rashid Chowdhury ( Ophthalmology), Dr. Md. Mustafizur Rahman (Nephrology), Dr. Md. Kazi Mazharul Islam (General, Laparoscopy and Hepatobilliary Surgery) , Dr. Md. Jahid Hasan (Medicine) and Mahmudul Hasan Raju of BRICM ( Senior Scientific Officer) for their inspiration, valuable advices, supervision and tremendous support. I also express my heartfelt thanks to all the health care workers of Department of ENT \& Head Neck Surgery and Dept. of Virology of DMCH, scientists and other staffs of Bangladesh Reference Institute of Chemical Measurements (BRICM) for their kind support and active participation during the research work.

Authors Contribution Arefin MK planned, designed the article; prepared \& revised the manuscript and finally submitted it.

\section{Declarations}

Conflict of interest There is no competing interest.

Ethical Approval Not applicable.

Informed Consent All of the patients or their attendants gave written informed consent to participate in the study willingly without any prejudice.

\section{References}

1. Khalil MM, Alam MM, Arefin MK et al (2020) Role of personal protective measures in prevention of COVID-19 spread among Physicians in Bangladesh: a multicenter cross-sectional comparative study. SN ComprClin Med. https://doi.org/10.1007/s42399-020-00471-1
2. Eggers M, Koburger-Janssen T, Eickmann M, Zorn J (2018) In vitro bactericidal and virucidal efficacy of povidone-iodine gargle/mouthwash against respiratory and oral tract pathogens. Infect Dis Ther 7:249-259

3. Kariwa H, Fujii N, Takashima I (2004) Inactivation of SARS coronavirus by means of povidone-iodine, physical conditions, and chemical reagents. Jpn J Vet Res 52:105-112

4. Arefin MK, Islam MZ, Shawrave MSA, AKMF Rahman. The Role of Povidone Iodine Mouthwash \& Nasal Spray or Drop or Inhalation for Protection (Prevention \& Treatment) of Health Care Professionals as Well as Patients and Reduction of Cross Infection during Current COVID-19 Pandemic Situation. . Intl J of Science \& Research (IJSR) ISSN: 2319-7064 .

5. Kirk-Bayley J, Challacombe S J, Sunkaraneni V S, Combes J. The use of povidone iodine nasal spray and mouthwash during the current COVID-19 pandemic may reduce cross infection and protect healthcare workers. 4 May 2020. Available at https://www.researchgate.net/publication/340320238_The_Use of_Povidone_Iodine_Nasal_Spray_and_Mouthwash_During_the _Current_COVID-19_Pandemic_May_Protect_Healthcare_Work ers_and_Reduce_Cross_Infection (accessed June 2020).

6. Mady LJ, Kubik MW (2020) Consideration of povidone-iodine as a public health intervention for COVID-19: utilization as personal protective equipment for frontline providers exposed in high-risk head and neck and skull base oncology care. Oral Oncol 105:104724

7. Khan MM, Parab SR, Paranjape M (2020) Repurposing 0.5\% povidone iodine solution in otorhinolaryngology practice in Covid 19 pandemic. Am J Otolaryngol 41(5):102618

8. Naqvi SHS, Citardi MJ, Cattano D et al (2020) Povidone-iodine solution as SARS-CoV-2 prophylaxis for procedures of the upper aerodigestive tract a theoretical framework. J of OtolaryngolHead Neck Surg 49:77. https://doi.org/10.1186/s40463-020-00474-x

9. Nagatake T, Ahmed K, Oishi K (2002) Prevention of respiratory infections by povidone-iodine gargle. Dermatology 204:32-36

10. Satomura K, Kitamura T, Kawamura T et al (2005) Prevention of upper respiratory tract infections by gargling: a randomized trial. Am J Prev Med 29:302-307

11. Gluck U, Martin U, Bosse B, Reimer K, Mueller S (2007) A clinical study on the tolerability of a Liposomal povidone-iodinenasal spray: Implications for further development. ORL 69(2):92-99. https://doi.org/10.1159/000097758

12. Arefin MK, Arafat MS, Talukder DC (2020) 'POLIDON' Approach-A Novel Solution for the ENT \& Skull Base Surgeons in COVID-19 era. Indian J Otolaryngol Head Neck Surg. https://doi.org/10.1007/s12070-020-02201-4

13. Arefin MK, Uddin MB, Haque MM, Azam MG (2020) 'POLIDON' approach - a novel approach of mastoidectomy in the COVID-19 pandemic. On J Otolaryngol \& Rhinol 3(3):1-8

14. Arefin MK, Uddin MB, Islam MZ, Arafat MS (2020) Novel technique of tracheostomy in the era of novel coronavirus. IJISRT 5(5):1149-1154

Publisher's Note Springer Nature remains neutral with regard to jurisdictional claims in published maps and institutional affiliations. 